\title{
Observations of OB-stars at the former Leiden Southern Station ${ }^{\star}$
}

\author{
C. J. van Houten ${ }^{\star}$
}

Sterrewacht Leiden, PO Box 9513, 2300 RA Leiden, The Netherlands

Received 25 February 2000 / Accepted 4 December 2000

\begin{abstract}
About 700 stars, mostly OB-stars, were observed by the author at the former Leiden Southern Station at Hartebeespoortdam, South Africa, in the observing seasons 1965, 1968, 1969, 1974, 1977, 1978. Observations were made in the five channels of the Walraven photometric system. Due to weathering of the telescope mirror the $W$ channel gave no reliable results for the faintest stars $(m=11 \mathrm{mag})$; in these cases the $U-W$ colour index is not given. The change in sensitivity in the $V$ channel, supposedly having occurred in 1968, was not recognised.
\end{abstract}

Key words. techniques: photometric - stars: fundamental parameters

\section{Introduction}

The first program carried out with the $90 \mathrm{~cm}$ lightcollector and Walraven 5-colour photometer after the installation of the telescope in South-Africa was a photometry of all then known O-and early B-stars in the southern hemisphere. This program was carried out in 1959 and 1960 by Th. Walraven (van Houten et al. 1999). In the meantime the number of known OB-stars has increased considerably so that it appeared worth while to augment the older survey with a new, deeper survey in selected parts of the southern milky way. This program was started in 1965 by the present author using the list of Lyngå (1964) and an unpublished list by Dr. E. H. Geyer, then of the Heidelberg Observatory. These lists were later augmented by selected stars from the catalogue of Stephenson \& Sanduleak (1971). In total about 700 stars were observed. This material is published here, containing the results of the observing campaigns of the years 1965, 1968, 1969, 1974, 1977 and 1978. Almost all selected stars were observed three times, in three different nights, according to the advice given by Walraven \& Walraven (1960).

\section{The phometric system, comparison with Lub \& Pel (1977)}

The photometric system is defined by the photometric values of a number of stars, which will be denoted here as standard stars. They number 10 in the present publication and are listed in Table 1 together with their adopted colours. Since the photometry yields only differences of

\footnotetext{
* Table 5 is only available in electronic form at the CDS via anonymous ftp to cdsarc.u-strasbg.fr (130.79.128.5) or via

http://cdsweb.u-strasbg.fr/cgi-bin/qcat?J/A+A/369/527

** e-mail: vhouten@strw.leidenuniv.nl
}

Table 1. The standard stars

\begin{tabular}{rrrrrr}
\hline HD & $V$ & $V-B$ & $B-U$ & $U-W$ & $B-L$ \\
\hline 61068 & 0.472 & -0.057 & -0.009 & -0.020 & -0.014 \\
74575 & 1.289 & -0.053 & -0.018 & -0.007 & -0.022 \\
88015 & 0.202 & -0.044 & 0.095 & 0.005 & 0.021 \\
104337 & 0.652 & -0.060 & -0.007 & -0.014 & -0.016 \\
122980 & 1.018 & -0.062 & 0.026 & -0.013 & 0.002 \\
144470 & 1.174 & 0.002 & 0.001 & 0.001 & 0.002 \\
151515 & -0.121 & 0.081 & -0.009 & 0.031 & 0.014 \\
164402 & 0.450 & 0.009 & -0.037 & 0.002 & -0.017 \\
191091 & -0.159 & -0.005 & 0.322 & 0.066 & 0.111 \\
210934 & 0.575 & -0.038 & 0.225 & 0.044 & 0.062 \\
\hline
\end{tabular}

brightnesses, a zero point for each colour must be adopted. The zero point adopted here is both the brightness and colours of standard star HD 144470 as given in Walraven et al. $(1964,1977)$. As usual in the Walraven photometric system, the brightnesses are expressed in logarithms of intensities.

Eight of the ten standard stars were also used as standards by Lub \& Pel (1977). The differences Lub \& Pel minus van Houten are listed in Table 3. It is seen that the average differences are $0.01 \mathrm{mag}$ or less.

In 1965 part of these standard stars were not used, but instead of them three other stars were used as standard stars. They are listed in Table 2. The other standard stars used in 1965 were HD 88015, 104337, 151515, 191091 and 210934. It appeared that the photometric system was somewhat different in 1965, which is explained as due to small changes in efffective wavelengths. For that reason stars observed in 1965 are denoted by 65 in the remarks column of the main table (Table 5). 
Table 2. Additional standards used in 1965

\begin{tabular}{rrrrrr}
\hline HD & $V$ & $V-B$ & $B-U$ & $U-W$ & $B-L$ \\
\hline 123980 & 0.010 & 0.060 & 0.443 & 0.137 & 0.214 \\
164806 & 0.023 & -0.012 & 0.149 & 0.021 & 0.015 \\
178175 & 0.519 & -0.009 & 0.076 & 0.004 & 0.013 \\
\hline
\end{tabular}

\section{Extinction}

The extinction coefficients for the different channels were determined in the usual way, by observing stars near zenith and stars observed through large airmass, selected from the list of standard stars. It was shown by Walraven \& Walraven (1960) that those five extinction coefficients can be expressed by one variable parameter,

$K=K_{0}+q \cdot K_{1}$

$K$ being the extinction coefficient, $K_{0}$ and $K_{1}$ constants for each channel, and $q$ the variable parameter. The values for $K_{0}$ an $K_{1}$ as given by Walraven \& Walraven (1960) were corrected using the results of Lub \& Pel (1977). Ideally the five channels should yield the same value of $q$; in practice this was not the case. These differences are due to inaccuracies of the measurements, such as by variable extinction. The method used was to take an average value of the five parameters $q$, and with this value recompute the extinction coefficients. By means of the extinctions so obtained the brightnesses of the standard stars were extrapolated to the zenith. The differences with the catalogued brightnesses were practically constant for each channel and average values were used for the reduction of the program stars. It turned out that the extinction at the observatory site was very variable, so that for each extinction measurement different $q$-values were found. The extinction values actually used for the reduction of the program stars were obtained by interpolating between these $q$-values.

\section{4. $V$-colour variation}

Lub \& Pel (1977) discovered that the colour dependence of their $V$ channel was different from that found by Walraven \& Walraven (1977); its cause was ascribed to a high voltage accident with the power supply of the amplifier in 1968. Since the observations presented here were partly made before that date, and partly thereafter, this fact gives the opportunity to check this assumption. In the present material 16 stars were observed both in 1965 and later than 1968, and 10 stars both in 1968, but before the calamity and later than 1968. Comparison between the 1965 and 1968 values with those obtained later should give clarity of this issue. First it should be realised that the choice of the zero point of the photometry, the same for pre-calamity and post-calamity, causes the constant term in the Lub \& Pel relation to vanish. The relation to be tested thus becomes:

$$
(V-B)_{\text {new }}-(V-B)_{\text {old }}=-0.026(V-B)_{\text {old }}
$$

Table 3. Differences: Lub \& Pel minus van Houten for standard stars

\begin{tabular}{rrrrrr}
\hline HD & $V$ & $V-B$ & $B-U$ & $U-W$ & $B-L$ \\
\hline 61068 & 0.000 & 0.000 & 0.000 & -0.001 & 0.000 \\
74575 & -0.003 & -0.003 & 0.004 & -0.010 & 0.001 \\
88015 & 0.002 & -0.003 & -0.002 & 0.001 & -0.004 \\
104337 & -0.005 & -0.006 & 0.005 & -0.007 & 0.001 \\
122980 & -0.001 & -0.004 & 0.007 & -0.005 & 0.003 \\
144470 & 0.002 & -0.005 & 0.004 & -0.003 & 0.002 \\
164402 & 0.005 & -0.006 & 0.007 & -0.001 & 0.005 \\
210934 & -0.002 & -0.002 & 0.006 & -0.002 & 0.004 \\
average & 0.000 & -0.004 & 0.004 & -0.004 & 0.002 \\
\hline
\end{tabular}

and a similar relation for $V$. Here "new" means: after, and "old" before calamity. The $V$ brightness, however, might possibly be influenced by small variations which do not influence its $V-B$ index; therefore only $V-B$ was compared. The 16 stars observed in 1965 yielded: $\Delta(V-$ $B)=-0.003 \pm 0.001(\Delta$ defined as in $(2))$ and the 10 stars observed in 1968: $\Delta(V-B)=-0.001 \pm 0.003$. Both values are, within their limits of accuracy, virtually equal to zero. Therefore it was decided to omit transformation (2) for the 1965 and 1968 observations and to give all observations in one list, re-calculated to the Lub \& Pel values for the standards.

\section{Precision}

With three observations per star the $V$ brightnesses can be averaged, as well as the four colour indices. This yields three differences with respect to this average value, and an average difference without regard to sign; this latter value will be denoted here as the precision of the observation. The results of such comparisons are summarized in Table 4. Here "brightest stars" means: stars of about 7 mag, and "faintest stars" means: stars of about mag 11. It can be seen that the $U-W$ colour index of the faintest stars is very uncertain, which is caused by the increasing oxidation of the telescope mirrors compared to 1959-1960. In Table 5 values for $U-W$ are only given when the precision (as defined above) is better than 0.016. Even this limit is not sufficient for a successful use of the spectral classification scheme developed by Walraven \& Walraven (1960). It is advised to omit the $U-W$ colour index altogether, except for the brightest stars. An attempt was made to partly counteract this dependence of accuracy on magnitude by increasing the integration time of one measurement for fainter stars. The usual integration time was $30 \mathrm{~s}$, but, depending on the brightness, it was increased to 2 min for the faintest stars.

\section{The main table}

Table 5 contains the measurements of the OB-stars observed in the years 1965, 1968, 1969, 1974, 1977 and 1978. 
Table 4. Accuracy of observations $(\Delta \log I)$

\begin{tabular}{lll}
\hline brightness or colour & brightest stars & faintest stars \\
\hline$V$ & 0.004 & 0.005 \\
$V-B$ & 0.002 & 0.004 \\
$B-U$ & 0.003 & 0.012 \\
$U-W$ & 0.005 & 0.040 \\
$B-L$ & 0.002 & 0.008 \\
\hline
\end{tabular}

Some stars are included which are not OB-stars; this is mostly a result of misidentifications. The listed values are mostly an average of three observations, if this number is different, it is mentioned in the column: obs. The stars are identified by their HD or CPD number, or, for those stars that do not have an HD or CPD number, by their number in the Lyngå (1964) and Stephenson \& Sanduleak (1971) catalogue. The latter are denoted with their Lyngå or Stephenson \& Sanduleak number, preceded with L or S respectively. As usual in the Walraven photometric system, the brighnesses are expressed in $\log I$.
Acknowledgements. Many thanks are due to Dr. E. H. Geyer for making a list of OB-stars available to the author, which was a very welcome addition to the lists of Lyngå and Stephenson \& Sanduleak. Mr. W. van Vliet and Dr. R. A. Windhorst are thanked for assistance in these observations. Mr. D. F. Stevenson took good care for a proper functioning of the instruments, which is hereby gratefully acknowledged.

\section{References}

van Houten, C. J., Walraven, Th., \& Walraven, J. H. 1999, A\&AS, 137, 113

Lub, J., \& Pel, J. W. 1977, A\&A, 54, 137

Lyngå, G. 1964, Lund Medd. Ser. II, 141

Stephenson, C. B., \& Sanduleak, N. 1971, Publ. Warner and Swasey Obs., 1, 1

Walraven, T., \& Walraven, J. H. 1960, Bull. Astr. Inst. Neth., 15,67

Walraven, T., \& Walraven, J. H. 1977, A\&AS, 30, 245

Walraven, J. H., Tinbergen, J., \& Walraven, T. 1964, Bull. Astr. Inst. Neth., 17, 520 